\title{
MODELLING TURBULENT PREMIXED COMBUSTION IN THE INTERMEDIATE STEADY PROPAGATION REGIME
}

\author{
Vladimir L. Zimont \\ CRS4, Sesta Strada Ovest, Z. I. Macchiareddu I-09010 Uta (Ca), Italy \\ Fernando Biagioli \\ Alstom Gas Turbines Ltd, Thorngate House, LN2 5DJ, Lincoln, UK
}

\begin{abstract}
Numerical simulation and comparison with standard experimental data of turbulent premixed combustion occurring at large Reynolds and moderately large Damköhler numbers (a situation which is typical in industrial burners) have been presented. The simulation has been performed in the framework of the Turbulent Flame-speed Closure (TFC) combustion model, developed in [1]-[4], which makes use of a theoretical expression for the turbulent combustion velocity for the closure of the progress variable transport equation. This model is based on the concept of Intermediate Steady Propagatioin (ISP) regime of combustion in real combustors, i.e. when the turbulent flame propagates with equilibrium turbulent flame speed but has flame brush thickness growing according to the turbulent dispersion law. These ISP flames precede usually analysed 1-D stationary flames and from the theoretical point of view they are in fact intermediate asymptotic of the combustion process between the period of formation of developed turbulent flames and 1-D stationary flames. Numerical results of turbulent premixed combustion in a two-dimensional planar channel at parameters that correspond to real industrial combustors have been compared with corresponding standard experimental data on a high speed turbulent premixed flame [9]. Finally, it has been explained in the framework of the TFC combustion model that "countergradient diffusion", i.e. the necessity to use a negative effective diffusion coefficient to describe experimental heat
\end{abstract}


and progress variable fluxes inside the flame, is an inherent feature of turbulent premixed flames and is connected with direct dependence of the second order velocity-scalars correlation on combustion. It has been shown that the existence of the countergradient diffusion phenomenon is not in contradiction with the actual increasing of the flame brush width.

Keywords: turbulent premixed combustion, turbulent flame speed, countergradient diffusion. 


\section{Introduction}

Turbulent premixed combustion in industrial burners occurs at large Reynolds numbers ( $R e>R e_{c r}$ where $R e_{c r}$ is the critical Reynolds number of the flow) where turbulence has a developed fine scale structure, and at relatively large Damkölher numbers $(D a>>1)$. In this case we have the flamelets combustion mechanism; chemical reactions will therefore complete in very thin layers (flamelet sheets), typically of width comparable to the smallest turbulent scales and much smaller than the integral length scale of turbulence.

The thin reacting flamelets will be highly wrinkled by small scale turbulent vortices (with size nevertheless significantly larger than the flamelet thickness); these have significant influence on combustion as they control the flame surface area and therefore the volumetric burning rate of the fresh fuel/air mixture with respect to an equivalent laminar flame. On the other hand, fine scale turbulence - with vortices of smaller and comparable size than the laminar flamelet thickness - controls the flamelets internal structure which may be thickened by this fine scale turbulence. At the same time flamelets stretch by turbulence can reduce the width of these flamelets incorporating fine scale vortices; therefore, generally speaking, the actual width of these flamelets thickened by fine scale turbulence can be even less that the width of an unstretched laminar flame (but obviously larger than the correspondent stretched laminar flame width). In any case the flamelets width is of the order of the unstretched laminar flame width and much less than the integral turbulence length scale, i. e. flamelets are thin.

Several modelling approaches have been proposed in literature so far to deal with turbulent premixed combustion. Most of them are based on laminar flamelet assumption, i.e. the turbulent flame viewed as an ensemble of wrinkled thin interfaces separating the unburnt mixture from the burnt one and having the structure of a laminar flame; the most famous is the model based on the Bray-Moss-Libby formalism [10, 11].

Another philosophy is used in probability density function methods. These use instead a chemical source term obtained from a given reaction mechanism; the effect of turbulent fluctuations on the average chemical production rates is accounted for by introducing a composition probability density function. This is often calculated by assuming a given shape $[12,13]$ generally parametrised with respect first and second order statistical moments 
of the independent thermo-chemical scalars or from a modelled transport equation generally solved via a Monte Carlo method [14, 13].

In all these cases the average rate at which reactants and products are mixed on the molecular scale has to be modelled; this is generally assumed to be proportional to the dissipation rate of turbulent kinetic energy, an assumption which is questionable in the case of flamelet combustion where simple analysis shows the scalar mixing rate to be directly dependent also from chemistry.

A substantially different modelling approach is the one based on the solution of a modelled transport equation for a flame surface density function [15]. According to this approach, when combustion occurs in the laminar flamelet regime, the volumetric rate of reactants consumption is proportional to the laminar flame speed times the flame surface area for unit of volume; the first of these quantities is generally calculated in a pre-processing stage while the average flame surface area is calculated from a modelled transport equation. The critical term of this modelled transport equation is the dissipation term which represents the reduction of flame surface area by the flamelet propagation mechanism.

According to the theoretical estimations $[1,2,3]$, which forms the basis of the TFC combustion model [1,4], turbulent premixed flames at the real Reynolds and Damköhler numbers typical of industrial burners are characterised by a) a flame structure composed by thin flamelets which may not be laminar but instead slightly (2-4 times) thickened by small scale turbulence, b) a burning rate of fresh reactants for unit of flame cross sectional area (turbulent flame speed) which is controlled by the flamelet sheet area and instantaneously adapts to a local equilibrium value, $\mathrm{c}$ ) at practically constant turbulent combustion velocity $U_{t}$ (in accordance with a. and b.), a flame brush width which is nevertheless growing in thickness according to the turbulent dispersion law.

Within the framework of the TFC combustion model we analyse the phenomenon of "countergradient diffusion", i. e. the situation when the direction of the averaged flux of heat or the progress variable is the same as the gradient of the average of these parameters. This phenomenon is well known from measurements in turbulent premixed flames $[5,6]$ and numerical simulations based on different modelling approaches confirm this results $[7,8]$. 
We analyse in this paper turbulent premixed flames with increasing flame brush width. Therefore, it is significant to clarify why this increase in flame brush width and "countergradient diffusion" are not in contradiction and that a positive turbulent diffusion coefficient controls the flame brush width. We think in fact that the term "countergradient diffusion" given to this effect characterising turbulent premixed flames is connected with non-correct interpretation of these fluxes as diffusion fluxes. Obviously, analysis of these fluxes in terms of the turbulent diffusion coefficient results that for agreement with experiments this coefficient must be negative ("countergradient diffusion" therefore).

These fluxes instead are controlled both by turbulence and combustion and the "countergradient diffusion" phenomenon is mainly connected with gas expansion due to heat release in flamelets while the flame brush width is controlled by real physical turbulent diffusion coefficient which can be estimated using, for example, the " $k-\epsilon "$ turbulence model. We therefore explain why the "countergradient diffusion" phenomenon and the increasing of the flame brush width are not in contradiction and what is the turbulent diffusion coefficient that is responsible for controlling the flame brush width.

In the paper we present the results of numerical simulation of experiments on $\mathrm{CH}_{4}$-air high velocity premixed combustion in a two-dimensional planar channel performed by Moreau [9]. For the same experiments and within the framework of the TFC combustion model we also give quantitative estimation of the "countergradient diffusion" effect.

\section{Analysis of turbulent premixed combustion in indus- trial combustors}

\subsection{Premixed combustion mechanism in industrial burners}

The instantaneous structure of a turbulent premixed flame depends on the relative value between quantities characterising a laminar premixed flame on one side (flame thickness $\delta_{L}$ and speed $s_{L}$ ) and turbulence on the other (turbulent intensity $u^{\prime}$ and integral length scale $\left.l_{t}\right)$

Several combustion regimes can be identified in this way and reported in a spectral diagram 
(The Borghi diagram [16]). In the case, for example, of laminar flame thickness $\delta_{L}$ smaller than turbulent Kolmogorov length scale $\eta$ and laminar flame speed $s_{L}$ smaller than the turbulence intensity $u^{\prime}$, the turbulent flame keeps locally its laminar flame structure and is highly wrinkled by turbulence.

A second situation occurs when the laminar flame thickness $\delta_{L}$ is larger than the Kolmogorov length scale but smaller than the integral length scale of turbulence $l_{t}$. In this case the turbulent vortices falling within the flamelets (more correctly falling within the preheat zone of the flamelets as the instantaneous reaction zone can be thinner than the Kolmogorov microscale $\eta$ ) will increase the internal diffusion coefficient between reactants and products determining an increase in flamelet thickness and local propagation velocity. According to the theoretical estimations in [2] the thickening and accelerating processes controlled by turbulent vortices in the Kolmogorov inertial interval stop when a new equilibrium between convection, diffusion and chemical reactions is reached inside the flamelet. This in fact means that the successive involvement of larger and larger vortices inside flamelets with successive increase of the flamelets width as consequence of such involvement has a limit; the final flamelet width corresponding to this limit is only several times larger than the one of the very thin laminar flamelet. Such increasing of the flamelet width therefore cannot transform the flame surface combustion mechanism into the volume combustion one where the instantaneous heat release is distributed in space similarly to the combustion process in stirred reactors with possible non-complete molecular mixing, a situation which is in fact the base of many combustion models (it would take place if vortices of all sizes had been successively involved in the reaction zone).

It will be assumed hereafter that industrial premixed combustion occurs in the regime of thin flamelets where for thin flamelets we mean flamelets which are thin with respect to the integral length scale of turbulence.

In our combustion model flamelets are thicker than laminar flamelets at the same conditions due to the influence of the fine scale vortices from the inertial interval on the transfer processes inside them.

The latest experimental data confirm this conclusion: the paper [17] demonstrates (through 
measurements of the instantaneous temperature gradients) that flamelets thickness is slightly larger than stretched laminar flames thickness (though smaller than the the thickness of the normal 1-D laminar flame); the results of the paper [18] (that seems more correct from the methodological point of view as the flamelets width was estimated from instantaneous temperature distributions) shows that experimental flamelet thickness is significantly (3-5 times) larger than it is for the normal laminar flame.

Broadening effect was demonstrated also in [19] by analysing 3-D gradient measurements of a progress variable. This effect was more severe at increasing of the Reynolds number.

\subsection{Development stages of a turbulent premixed flame}

It will be considered here that the turbulence intensity $u^{\prime}$ is sufficiently larger than the laminar flame speed $s_{L}$ (more correctly than the flamelet combustion speed $s_{L}^{*}$ which will be defined later), a situation which generally occurs in gas turbines combustors (typical values for the turbulence intensity are in the range of $u^{\prime}=5-10 \mathrm{~m} / \mathrm{s}$ while the laminar flame speed is in the range of $0.5-1.0 \mathrm{~m} / \mathrm{s}$ for methane).

It is very useful to analyse the problem starting from the simple case of a 1-D freely propagating turbulent premixed flame. Consider therefore a planar 1-D freely propagating laminar premixed flame that at time $t=0$ is interested by a homogeneous turbulent field with intensity $u^{\prime}$ and length scale $l_{t}$. The following four development stages can be postulated:

1. $0<t<t_{1} \sim \tau_{c h}$. Flamelet thickening. In the case of $\eta<<d_{L}<<l_{t}$, small scale turbulence will penetrate inside the thin flame preheating zone increasing the internal diffusion coefficient between products and reactants; as a consequence this will determine an increase of the flamelet thickness and flamelet speed until new equilibrium between convection, diffusion and chemical source term will be established. Using dimensional analysis, analogously to the case of laminar combustion, we obtain:

$$
s_{L}^{\star} \simeq \sqrt{\lambda^{\star} / \tau_{c h}} \quad d_{L}^{\star} \simeq \sqrt{\lambda^{\star} \tau_{c h}}
$$

where $\tau_{c h}$ is a characteristic chemical time scale and $\lambda^{\star}$ is the turbulent thermal diffusivity coefficient determined by turbulent scales falling within the flamelet which may 
be approximated as

$$
\lambda^{\star} \simeq u^{\prime \star} l^{\star} \simeq u^{\star \star} d_{L}^{\star}
$$

According to the process of turbulent kinetic energy cascade across the various turbulent scales we have:

$$
\frac{u^{\prime 3}}{l_{t}} \simeq \frac{u^{\star \star 3}}{d_{l}^{\star}} \Rightarrow u^{\prime \star} \simeq\left(\frac{d_{l}^{\star}}{l_{t}}\right)^{1 / 3} u^{\prime}
$$

Finally, using relations (1)-( 3 ) the following expressions are obtained:

$$
d_{l}^{\star} \simeq l_{t}\left(\frac{\tau_{c h}}{\tau_{t}}\right)^{3 / 2} ; \quad s_{L}^{\star} \simeq u^{\prime}\left(\frac{\tau_{c h}}{\tau_{t}}\right)^{1 / 2}
$$

which show that the smaller the Damkölher number $\left(D a=\tau_{t} / \tau_{c h}\right)$, the larger the final flamelet thickness and velocity. It is assumed that this process, which is governed by the smallest turbulent scales, will complete very quickly, typically in a time comparable to the chemical time scale $\tau_{c h}$, i. e. taking the same order of time that is necessary to develop laminar flames. Note that the relations $\eta<<d_{L}{ }^{\star}<<l_{t}$ and $u^{\prime}>>s_{L}{ }^{\star}$ transforms in terms of Damköhler and Reynolds numbers in [2]:

$$
R e^{3 / 4} D a^{-3 / 2} \geq 1>D a^{-1 / 2}
$$

which shows that for a given Reynolds number, the Damköhler number has to be larger than one (otherwise $s_{L}{ }^{\star}$ would be order of unity and no flame wrinkling will occur) and small enough for some turbulent scales to penetrate inside it (left inequality).

2. $0<t<t_{2} \sim \tau_{G}$. Flamelet wrinkling. The second stage is the wrinkling of the flamelet sheet by turbulence. Not all turbulent scales can effectively contribute to the wrinkling of the turbulent flamelet; in fact, those with a characteristic velocity which is smaller than the flamelet velocity $s_{L}{ }^{\star}$ will be spoiled by the flamelet propagation mechanism. The final equilibrium length scale of the smallest flamelet wrinkles (often called Gibson scale) will therefore be equal to the size of vortices whose velocity is equal to $s_{L}{ }^{\star}$ :

$$
\epsilon \simeq \frac{u^{\prime 3}}{l_{t}}=\frac{s_{L}^{\star 3}}{l_{G}} \Rightarrow l_{G} \simeq l_{t}\left(\frac{s_{L}}{u^{\prime}}\right)^{3}
$$

and the corresponding time scale given by:

$$
\tau_{G}=\frac{l_{G}}{s_{L}{ }^{\star}} \simeq \tau_{t}\left(\frac{s_{L}^{\star}}{u^{\prime}}\right)^{2}
$$


It should be particularly emphasised that the flamelet sheet area is controlled mainly by small scale sheet wrinkles with size that are of the order of $l_{G}$ and therefore the time that is necessary for forming turbulent flame with constant turbulent combustion velocity

$$
U_{t}=s_{L}^{\star} \frac{\bar{A}}{A_{0}},
$$

(where $A / A_{0}$ is the instantaneous flamelet sheet area for unit of cross sectional area) is of the order of $\tau_{G}$. Considering the case of an industrial gas turbine combustor with length equal to $0.5 \mathrm{~m}$, average velocity $50 \mathrm{~m} / \mathrm{s}, l_{t}=0.02 \mathrm{~m}$ and $u^{\prime}=5 \mathrm{~m} / \mathrm{s}$, $s_{L^{\star}}=1 \mathrm{~m} / \mathrm{s}$ we have a residence time of $\tau_{r}=0.01 \mathrm{sec}$ and $\tau_{G}=0.00016 \mathrm{sec}$; it follows $\tau_{G} / \tau_{r}=0.016$, i. e. we have practically equilibrium turbulent combustion velocity from the beginning.

At the same time the flame brush width is controlled by large scale turbulent vortices with sizes of the order of $l_{t}$. This explains (as we see below) the existence in real industrial combustors of turbulent flames with increasing flame brush width and practically constant flame velocity.

3. $0<t<t_{3} \sim \tau_{s t}$ Thickening of the turbulent flame brush. The effect of large scale turbulence with $u^{\prime}>>s_{L}{ }^{*}$ is to increase the width of the turbulent flame brush according to the turbulent dispersion law; in fact, the averaged transport of the flamelet sheet elements due to turbulent pulsations (turbulent diffusion) is much larger during this time interval than the averaged transport because of the local propagation velocity $s_{L}^{*}$. At the conditions characterising combustion in industrial burners this increasing would take place on a time scale which is much larger than the residence time, i.e. in real combustors we have turbulent flames with increasing width.

In fact, in a frame of reference moving at velocity $U_{t}=$ const, the turbulent flame will appear in a given position with brush thickening with time according to $\Delta_{F} \simeq \sqrt{2 D_{t} t}$ where $D_{t} \simeq u^{\prime} l_{t}$ is the turbulent diffusion coefficient. It means therefore that during this period the flame constitutes in fact a turbulent mixing layer with increasing width moving with the constant velocity $U_{t}$. 
4. $t>t_{3} 1-D$ stationary combustion front. The flame brush thickening at velocity $d \Delta_{F} / d t$ is counteracted by the flamelet propagation at velocity $s_{L}{ }^{\star}$. Constant flame brush thickness therefore is obtained on a time scale given by:

$$
\left.\frac{d \Delta_{F}}{d t}\right|_{\tau_{s t}}=\sqrt{\frac{D_{t}}{\tau_{s t}}}=s_{L}{ }^{\star} \Rightarrow \tau_{s t} \sim\left(\frac{u^{\prime}}{s_{L}{ }^{\star}}\right)^{2} \tau_{t} .
$$

At $t>t_{3}$ flames would have the structure close to asymptotical 1-D (at $\left.t \rightarrow \infty\right)$ stationary combustion front. As we mentioned before this time at $u^{\prime} / s_{L}^{*}>>1$ is much larger than $\tau_{t}$ and the residence time in the combustor.

Hence industrial turbulent premixed combustion takes place in flames with equilibrium (stationary) combustion velocity, that depends on the turbulence and physico-chemical parameters of the mixture, and increasing flame brush thickness according to the turbulent diffusion law (i. e. with the thickness that does not depend directly on chemistry). We call these flames Intermediate Steady Propagation Flames (ISP Flames) and this combustion regime Intermediate Steady Flame Propagation Regime.

According to our estimations 1-D stationary combustion flames, often considered in theoretical investigations, (constant $U_{t}$ and $\Delta_{f}$ ) are not typical for industrial premixed combustion as the actual residence time in gas turbine combustors is much less than the time that is necessary to form flames which have the structure of the 1-D stationary combustion front (the general picture can be $2-\mathrm{D}$ or $3-\mathrm{D}$ but the flame structure remains similar to the 1 D stationary combustion front). In other words combustion is brought to completion well before forming the flame that has the structure of the 1-D stationary combustion front.

The following picture emerges from the previous considerations: in the case of large Damköhler and Reynolds number combustion the period of the turbulent flame speed transient behavior (the period to form a developed flame sheet structure which is characterised by fast increase of the combustion velocity) is of no importance (the turbulent flame speed will adapt vary rapidly to a local equilibrium value); at the same time the flame brush will grow in thickness along all the combustion chamber according to the turbulent dispersion law, the compensation of such thickening by local flamelet propagation being not relevant because local flamelet propagation becomes effective on a time scale which is much larger than the residence time 
in the combustor. The postulation of the existence of such combustion regime in industrial premixed combustion is extremely helpful for the modelling process as it a priori shows that some of the physical mechanisms forming and controlling the structure and properties of 1-D stationary combustion fronts — which are very difficult to account for-are not important and they do not need to be modelled.

It should be particularly emphasized that the properties and controlling physico-chemical mechanisms of the ISP flames and 1-D stationary flames are quite different. The turbulent combustion velocity of the 1-D stationary front $U_{t}^{s t}$ at strong turbulence $\left(u^{\prime}>>s_{L}{ }^{*}\right)$, in accordance with Damköhler [20], Shchelkin [21] and Zel'dovich [22] view of turbulent combustion, is $U_{t}^{s t} \sim u^{\prime}$, i.e. does not depends on chemistry. In accordance with Zel'dovich concept of leading points (these are the product volumes most advanced by turbulence in the fresh mixture), the turbulent combustion velocity is controlled by the velocity of these points which form the frontal edge of the flame front. In this case the flamelet sheet area will conform to this velocity in order to "fulfill" (8), which means that the averaged flame width depends on $s_{L}^{*}, d_{l}^{*}$ and the turbulence parameters.

For ISP flame the situation is quite different: the turbulent combustion velocity $U_{t}$ (the fresh mixture volumetric consumption rate) is controlled directly by $s_{L}^{*}$ and actual flamelets sheet area (they are controlled by turbulence and physico-chemical properties of mixture) and it in fact does not depends on the velocity of the front edge (that is obviously larger than $U_{t}$ in flames with increasing width).

\section{Modelling equation. The countergradient diffusion effect.}

We will consider first the problem of non-stationary 1-D turbulent premixed flame. The equation of the TFC combustion model in terms of the progress variable is the following one:

$$
\frac{\partial(\bar{\rho} \tilde{c})}{\partial t}+\frac{\partial(\bar{\rho} \tilde{u} \tilde{c})}{\partial x}=\frac{\partial}{\partial x}\left(\bar{\rho} D_{t} \frac{\partial \tilde{c}}{\partial x}\right)+\rho_{u} U_{t}\left|\frac{\partial \tilde{c}}{\partial x}\right|
$$


where $D_{t}$ is the physical positive turbulent diffusion coefficient (we have used in this work a standard $k-\epsilon$ model to calculate it), and $U_{t}$ in the modelled source term is the turbulent combustion velocity. In our simulations we have used for $U_{t}$ the theoretical expression derived in [2] using the Kolmogorov methodology; this corresponds to thickened flamelets because of fine scale turbulence of the inertial interval and to equilibrium small scale wrinkled structure of the random flamelet sheet which controls the flame surface area. We will discuss this expression in the next paragraph. The well known unclosed equation of this problem is:

$$
\frac{\partial(\bar{\rho} \tilde{c})}{\partial t}+\frac{\partial(\bar{\rho} \tilde{u} \tilde{c})}{\partial x}=\frac{\partial\left(-\bar{\rho} \widetilde{u}^{\prime \prime} c^{\prime \prime}\right)}{\partial x}+\bar{W}
$$

where $\bar{W}$ is the real chemical source term. It is a well established fact that the transport term $-\bar{\rho} \widehat{u^{\prime \prime} c^{\prime \prime}}$ has "countergradient" nature, i.e. in order to describe it in terms of an effective diffusion coefficient this coefficient must be negative. This is why first of all we will discuss this apparent contradiction and we will show how to describe this phenomenon in the framework of the TFC combustion model.

In a frame of reference moving with the turbulent flame speed $U_{t}$ equation (11) becomes:

$$
\bar{\rho} \frac{\partial \tilde{c}}{\partial t}+\rho_{u} U_{t} \frac{\partial \tilde{c}}{\partial x}=-\frac{\partial\left(\bar{\rho} \widetilde{u^{\prime \prime} c^{\prime \prime}}\right)}{\partial x}+\bar{W}
$$

with $\bar{\rho} \tilde{u}=\rho_{u} U_{t}=$ const for mass conservation.

As already explained in the previous section for $t<<\tau_{s t}$ (ISP flames) the turbulent flame brush will increase its thickness with time according to the turbulent dispersion law.

A modelling equation for such a scenario is:

$$
\bar{\rho} \frac{\partial \tilde{c}}{\partial t}=\frac{\partial}{\partial x}\left[\bar{\rho} D_{t} \frac{\partial \tilde{c}}{\partial x}\right]
$$

where $D_{t}$ is the turbulent diffusion coefficient.

Using this expression in equation ( 12 ) we obtain:

$$
\rho_{u} U_{t} \frac{\partial \tilde{c}}{\partial x}=\bar{W}+\frac{\partial}{\partial x}\left[-\bar{\rho} \widehat{u^{\prime \prime} c^{\prime \prime}}-\bar{\rho} D_{t} \frac{\partial \tilde{c}}{\partial x}\right]
$$

which shows that the term on the 1.h.s. may be used to model at the same time the progress variable source term $\bar{W}$ and the difference between the second order velocity-progress variable correlation and the real turbulent transport term. 
We assume in our estimations that the averaged source term in equation (14) is proportional to the probability to find the flamelet at a given position $p_{\text {flam }}(x, t)$; this probability is related to the probability of finding products $P_{b}$ at the given position by the relation:

$$
P_{b}(x, t)=\int_{-\infty}^{x} p_{\text {flam }}(x, t) d x \Rightarrow p_{\text {flam }}(x, t)=\frac{\partial P_{b}}{\partial x}
$$

Note also that

$$
\bar{c}=P_{b} 1+\left(1-P_{b}\right) 0=P_{b}
$$

Therefore we can write:

$$
\bar{W}=\operatorname{const} \frac{\partial \bar{c}}{\partial x}
$$

where the constant is equal to $\rho_{u} U_{t}$ as can be shown by integrating equation (14) from $-\infty$ to $+\infty$.

Using eqn. ( 13) in eqn. (14) the following expression for the space derivative of the second order velocity-progress variable correlation is obtained:

$$
-\frac{\partial\left(\bar{\rho} \widetilde{u^{\prime \prime} c^{\prime \prime}}\right)}{\partial x}=\rho_{u} U_{t} \frac{\partial(\tilde{c}-\bar{c})}{\partial x}+\frac{\partial}{\partial x}\left[\bar{\rho} D_{t} \frac{\partial \tilde{c}}{\partial x}\right]
$$

which integrated from $-\infty$ to $x$ yields:

$$
-\bar{\rho} \widetilde{u^{\prime \prime} c^{\prime \prime}}=\rho_{u} U_{t}(\tilde{c}-\bar{c})+\bar{\rho} D_{t} \frac{\partial \tilde{c}}{\partial x}
$$

This relation shows that the second order Favre correlation between the progress variable and velocity fluctuations is composed of two contributions:

a) a real turbulent transport term (modelled here with an eddy diffusivity assumption) which is responsible for the thickening of the flame brush always observed in experiments;

b) a contribution which is proportional to the integral of the difference between $\rho_{u} U_{t} d \tilde{c} / d x$ and the Reynolds averaged chemical source term $\bar{W}=\rho_{u} U_{t} d \bar{c} / d x$. This term is different from zero and can be expressed as function of $\tilde{c}$ by the relation:

$$
\rho_{u} U_{t}(\tilde{c}-\bar{c})=\rho_{u} U_{t} \tilde{c}(1-\tilde{c}) \frac{1-\tau_{\rho}}{1+\tilde{c}\left(\tau_{\rho}-1\right)}
$$


where $\tau_{\rho}=\rho_{u} / \rho_{b}$ is the ratio between the density of the fresh mixture and the hot burnt gases. This term is plotted in figure 1 versus $\tilde{c}$ and, if related to an eddy diffusivity assumption, it yields a negative diffusion coefficient such that very often it is said that "countergradient diffusion" is present in turbulent premixed combustion.

The global effect in the general case can correspond to the "gradient" or to "countergradient diffusion" (it depends on relationship among these two terms). To further clarify this point we emphasise that the velocity-progress variable correlation can be always expressed directly in terms of the conditional averaged velocities for the unburnt and burnt mixtures (respectively $\bar{u}_{u}$ and $\bar{u}_{b}$ )

$$
-\bar{\rho} \widetilde{u^{\prime \prime} c^{\prime \prime}}=\bar{\rho}\left(\bar{u}_{u}-\bar{u}_{b}\right) \tilde{c}(1-\tilde{c})
$$

For a 1-D stationary combustion front we have $\bar{u}_{u}(x=-\infty)=U_{t}^{s t}$ and $\bar{u}_{b}(x=+\infty)=$ $\left(\rho_{u} / \rho_{b}\right) U_{t}^{s t}$. As $\rho_{u}>\rho_{b}$ obviously $\bar{u}_{u}<\bar{u}_{b}$ and we always would have "countergradient diffusion" that is consequently an inherent property of 1-D stationary flames and is related to the expansion of the gas because of combustion.

Our estimation (20), which represents the upper bound of this effect, obviously corresponds to the assumption that $\bar{u}_{u}(x)=u(x=-\infty)=U_{t}^{s t}$ and $\bar{u}_{b}(x)=u(x=+\infty)=\left(\rho_{u} / \rho_{b}\right) U_{t}^{s t}$. The real "countergradient diffusion" effect in a 1-D front is obviously smaller (by approximately one third compared to results reported in $[5,6,7,8])$.

The main reason for this is that gas expansion results in 1-D flame decreasing of pressure; therefore in real flame $\bar{u}_{u}(x)>u(x=-\infty)$ and $\bar{u}_{b}(x)<u(x=+\infty)$. A second physical mechanism that reduces (we presume not so significantly as the first one) the conditional velocities difference is shock interraction between hot and cold volumes when hot volumes moving in lateral direction penetrate in cold gas and cold volumes penetrate in hot gas.

For ISP flames under investigation here, in the case of relatively small density difference between unburned and burn gas and physical turbulent diffusion coefficient sufficiently large, we will have the "gradient diffusion" in accordance with (19) (i.e. the first term on the r.h.s. is relatively small); in the opposite case we will have the "countergradient diffusion". But in our model only the real physical turbulent diffusion coefficient controls the increasing of flame brush width (the same coefficient would control, for example, the mixing of a 
nonuniform concentration of some passive addition introduced through the flame). The "countergradient" part ( 20 ) of the second order correlation $-\bar{\rho} \widetilde{u} c^{\prime \prime}$ does not affect the flame brush width of the ISP flames (and the mixing of nonuniform concentration of passive addition across the flame passive).

It means that our model source term given by eqn. (14) is the sum of the real combustion source term and the "countergradient diffusion" term given by (20). To separate them we use in fact a model for heat release given by (17) that in some sense corresponds to the upper estimation of the countergradient diffusion.

Of course, as already mentioned, there are other mechanisms which might contribute to countergradient diffusion (nonuniformity of flamelets structure in space and corresponding nonuniformity of the flamelets combustion velocity and so on), but we believe they are not so significant factors in comparison with gas expansion due to heat release.

\section{Extension to the general case}

Consider now the general 3D case; the progress variable transport equation for this case is given by (steady flow assumed):

$$
\nabla(\bar{\rho} \tilde{\mathbf{u}} \bar{\rho} \tilde{\mathbf{u}} \tilde{c})=\nabla \cdot\left(-\bar{\rho} \widetilde{\mathbf{u}^{\prime \prime} c^{\prime \prime}}\right)+\bar{W}
$$

This equation can be written locally in a frame of reference attached to the flame as:

$$
\bar{\rho} \mathbf{u} \cdot \nabla \tilde{c}=\frac{\partial}{\partial n}\left(-\bar{\rho} \widehat{u_{n}^{\prime \prime} c^{\prime \prime}}\right)+\frac{\partial}{\partial \tau}\left(-\bar{\rho} \widehat{u_{\tau}^{\prime \prime} c^{\prime \prime}}\right)+\bar{W}
$$

where $n$ and $\tau$ are respectively the normal and tangential direction relative to the flame.

The terms at the r.h.s., accounting for relation (19), can be reformulated in this equation in the following way:

$$
\underbrace{\bar{W}+\frac{\partial}{\partial n}\left[\left(-\bar{\rho} \widehat{u_{n}^{\prime \prime} c^{\prime \prime}}\right)-\bar{\rho} D_{t} \frac{\partial \tilde{c}}{\partial n}\right]}_{\rho_{u} U_{t} \partial \tilde{c} / \partial n}+\frac{\partial}{\partial \tau} \underbrace{\left[-\bar{\rho} u_{\tau}^{\prime \prime} c^{\prime \prime}\right]}_{\bar{\rho} D_{t} \partial \tilde{c} / \partial \tau}+\frac{\partial}{\partial n}\left[\bar{\rho} D_{t} \frac{\partial \tilde{c}}{\partial n}\right]
$$

The final modelled equation for the progress variable can be set therefore in the form:

$$
\nabla \cdot(\bar{\rho} \tilde{\mathbf{u}} \hat{c})=\nabla \cdot \nabla\left(\bar{\rho} D_{t} \nabla \hat{c}\right)+\rho_{u} U_{t}|\nabla \hat{c}|
$$


This equation states that the flame will locally stabilise with respect to the main flow direction according to an angle which gives a normal flow velocity component equal to the turbulent flame speed. Furthermore the flame will grow in thickness according to the turbulent dispersion law. Note finally that the source term in the progress variable equation ( 25) at the same time accounts for the real source term and the "countergradient" diffusion term which therefore doesn't need to be modelled if we want to simulate the space distribution of the hydrodynamical and thermodynamical parameters and concentrations. But if we want to describe the real space distribution of the averaged combustion (averaged heat release intensity distribution) we must extract it from the model source term as it will be shown below.

The last step necessary to close the set of equations will be to evaluate the turbulent flame speed as function of significant physico-chemical parameters.

\section{Modelling of the turbulent flame speed}

In our calculation (quite similarly to previous works $[23,24]$ ) we used in (25) the theoretical expressions for the $U_{t}[2]$ based on the estimations in (8) of $s_{L}{ }^{*}$ and $\overline{A / A_{0}}$ according to the Kolmogorov methodology (assumption on the existence of equilibrium fine-scale turbulence controlling flamelets property and equilibrium small-scale flamelet sheet structure controlling sheet area). The expressions obtained for these two quantities are:

$$
\begin{aligned}
& \frac{s_{L}^{\star}}{\frac{A}{A_{0}}} \simeq u^{\prime} D a^{-1 / 2} \\
& \simeq a^{3 / 4}
\end{aligned}
$$

According to the first of these relations the local propagation velocity of the thickened flamelet increases with decreasing Damköhler number. This can be explained considering that a reduction in Damköhler number produces an increase in thickness of the flamelet; as a consequence the turbulent diffusion coefficient inside it increases and therefore also the local propagation velocity increases.

The increase of the flamelets propagation velocity (for example, due to an increase of the mixture temperature) produces decrease of the flamelet sheet area $A$ because of the increase 
in size of the small-scale flame wrinkles (Gibson scale); this will compensate in significant amount the increase of $s_{L}{ }^{*}$ and result in a weaker dependency of $U_{t}$ from chemistry in comparison with laminar flames. The final expression for the turbulent combustion velocity is as follows:

$$
U_{t} \simeq u^{\prime} D a^{1 / 4}
$$

The effect of large strain rate (large dissipation rate of the turbulent kinetic energy $\epsilon$ ) is to reduce the local flamelets velocities and even cause their extinction; this effect has been incorporated in the expression for $U_{t}$ similar to $[25,26]$ using a model developed by Bray [27]. We have used here a stretching factor $G$ which represent the probability for $\epsilon$ to be less than the critical extinction value $\epsilon_{c r}$. For $\epsilon>\epsilon_{c r}$ flamelets extinction takes place while for $\epsilon<\epsilon_{c r}$ the stretch-effect is ignored completely. Assuming a log-normal distribution for $\epsilon$ the stretching factor is given by:

$$
G=0.5 \operatorname{erfc}\left[-\frac{1}{\sqrt{2 \sigma}}\left(\ln \left(\epsilon_{c r} / \epsilon\right)+\frac{\sigma}{2}\right)\right]
$$

where erfc denotes the complementary error function, $\sigma=\mu \ln \left(l_{t} / \eta\right)$ the standard deviation ( $\mu=0.28$ being a constant). The final expression for $U_{t}$ is therefore given by:

$$
U_{t} \simeq G u^{\prime} D a^{1 / 4}
$$

Note that an accurate estimation of $\epsilon_{c r}$ is necessary to correctly account for the "bending" of $U_{t}$ in the dependence $U_{t}=f\left(u^{\prime}\right)$; at high turbulence intensity in fact increasing of $u^{\prime}$ results in decreasing of $U_{t}[4,25,26]$.

Some results on the validation of the model for the case of flames in spherical bombs with artificial generation of turbulence can be found in the papers $[4,25,26]$; application of the model to industrial premixed combustion can be found instead in ref. [24] and a preliminary application to the Moreau experiments considered here in ref. [23].

\section{Calculation of a high speed turbulent flame}

The test case selected for validation of the combustion modelling philosophy presented here is a 2D planar turbulent premixed flame experimentally studied by Moreau [9]. The combustor 
is sketched in figure 2 together with the inlet conditions used. This flame is in the regime of thickened flamelets.

Some discrepancy has been observed between the nominal input data and the experiments at $x=0.039 \mathrm{~m}$. For example the temperature of the pilot hot gases is rather low despite an equivalence ratio equal to unity; this could be due to heat losses in the pilot burner. In order to account for the different equivalence ratio characterising the pilot gases a second passive scalar has been introduced in the simulation; instantaneous mixing of burnt gases from the main fuel/air inlet with pilot hot gases is assumed (therefore no passive scalar variance equation is used). The composition of the pilot gases is assumed to be the equilibrium one at the fixed temperature of $2000 \mathrm{~K}$; the composition of the burnt fuel/air mixture is assumed to be the adiabatic equilibrium one.

By simple transformations it is possible to put equation ( 30 ) in the following form:

$$
U_{t}=A G u^{3 / 4} s_{L}{ }^{1 / 2} \lambda_{u}^{-1 / 4} l_{t}^{1 / 4}
$$

where the local turbulent velocity intensity $u^{\prime}$ and integral length scale $l_{t}$ can be obtained from the turbulent kinetic energy and its dissipation rate; in this expression $\lambda_{u}$ is the molecular heat transfer coefficient of the fresh mixture, $A$ is a modelling constant that have the universal value $A \simeq 0.5$ (with exception of $H_{2} /$ air flames were $A \simeq 0.6$ ) [4]. The molecular diffusion coefficient at $T=600 \mathrm{~K}$ (which is the inlet temperature of the fresh fuel/air mixture) is $\lambda_{u}=7.15 e-05 \mathrm{~m}^{2} / \mathrm{sec}$ and the laminar flame speed (which can be calculated using any flamelet code for freely propagating laminar flames) $s_{L}=1.1 \mathrm{~m} / \mathrm{s}$.

The simulation has been performed using a finite volume CFD solver based on the SIMPLE method. A standard $k-\epsilon$ model has been used to calculate turbulent viscosity. The computational domain has been discretised with a $80 \times 50$ mesh. Boundary conditions are shown in figure 2 (the definition used here for the turbulent kinetic energy dissipation is $\left.\epsilon=k^{3 / 2} / l\right)$.

This flame is substantially characterised by two important features as can be understood from the progress variable contours in figure 3 and the composition profiles at different axial section in figure 4 :

- spreading of the flame brush thickness from the inlet to the outlet; 
- inclination of flame brush with respect to the direction of the fresh mixture because of the turbulent flame speed.

The stretch factor $G$ depends on the value of the critical velocity gradient $g_{c r}$ which is difficult to determine with precision. A rough estimation of this value would be the inverse of the chemical time scale $\tau_{c h}^{-1}$; previous work [4] has shown an uncertainty between 50 and $150 \%$ of this value. The best agreement with experiment has been obtained here assuming $g_{c r}=0.6 / \tau_{c h}=10,000 \mathrm{sec}^{-1}$. Figure 3 also shows contours of the stretch factor. It should be observed that this is significantly smaller than unity in the large shear region between the pilot gas and unburnt mixtures inlets; this results in local suppression of combustion and in delay of propagation of the flame which is significant in the comparison with the experimental data. The good agreement with the experimental composition profiles shows that the turbulent burning velocity and spreading rate of the turbulent flame brush are reasonably well predicted. Figure 5 shows temperature and velocity profiles at different axial locations. The predicted temperature is about $200 K$ larger than the one experimentally observed a possible reason for the disagreement being heat losses at the wall for cooling (which are not accounted for by the combustion model). The velocity profiles are in good agreement with the experiments. Figure 6 shows the contours and profiles at two different locations across the flame brush of the model source term used in the progress variable equation $\rho_{u} U_{t}|\nabla \hat{c}|$ and of the chemical source term calculated according to the assumption $\rho_{u} U_{t}|\nabla \bar{c}|$. The figure clearly shows that the chemical source term is shifted in the front part of the flame with respect to the model source term; this, as already explained, is responsible for the "countergradient diffusion" mechanism. Finally, figure 7 and 8 show contours and profiles of gradient $\bar{\rho} D_{t} d \tilde{c} / d n$, countergradient $\rho_{u} U_{t}(\tilde{c}-\bar{c})$ and total $-\bar{\rho} u_{n}^{\prime \prime} c^{\prime \prime}$ (calculated according to the expression (19)); these figures give a graphic representation of the relation between the three fluxes: the total one being of "countergradient" type and given by the sum of a positive contribution (turbulent transport) and a negative one ("countergradient diffusion" related to gas expansion). 


\section{Conclusions}

It is well established that turbulent premixed combustion at large Reynolds and Damköhler numbers which are typical in industrial burners is characterised by the flamelet combustion mechanisms. Uncertainty may eventually still be present only on the flamelet structure: the question being if the flamelet is locally laminar or fine-scale turbulence and flow stretch have large effect on the flamelets structure, but, in general, this details cannot change the flamelets combustion mechanism.

We believe that only combustion models based on the realistic flamelet combustion mechanism can describe standard experimental data and give correct trends for variations of the physico-chemical parameters.

The use of the flamelets combustion mechanism gives opportunity to simplify the problem of industrial combustors simulation by eliminating from the combustion model the regime of 1-D stationary flame. Such opportunity is based on the assumption (strengthened by the theoretical estimations and simulation comparisons with the experimental data) that combustion is brought to completion long before the formation of flames with the structure of 1-D stationary flame occurs. It gives an opportunity to restrict the combustion simulation in industrial combustors at intensive turbulence (when $u^{\prime}>s_{L}^{*}$ ) using the combustion model which describes only the Intermediate Steady Propagation Regime, i.e. regime when combustion takes place in flames that propagate with equilibrium combustion velocity (in the sense that very quickly adapts to the local turbulence) but with thickness increasing according to the turbulent dispersion law.

The transient behavior of the turbulent flame speed (the formation of equilibrium small-scale wrinkled flamelet sheet structure controlling its area) doesn't need to be accounted for as equilibration occurs on a time scale (the Gibson time scale) which in the case of $u^{\prime} / s_{L}^{*}>>1$ is much smaller than the residence time in the combustor. At the same time the compensation of flame brush thickening by the local flamelet propagation occurs on a time scale which is much larger than the residence time; this effect (which yields a flame structure similar to 1-D stationary front) therefore doesn't need to be accounted for during the modelling process. The TFC model gives the opportunity to describe all experimental profiles of Moreau mea- 
surements using in fact only one empirical parameter $A=0.5$, that was defined from $\mathrm{V}$. Karpov standard experiments in the bombs at high artificially generated turbulence with $R e$ and $D a$ numbers corresponding to real premixed industrial burners. It means that the model can predict not only the angle of the flame in a chamber (controlled by $U_{t}$ ) but also profiles of several quantities across the flame, i. e. the flame structure. In fact we have used Moreau experimental data as the standard data to test the TFC model as an industrial combustion model. Earlier this model was used to simulate a real industrial premixed combustion burner [24].

The phenomenon of "countergradient diffusion" has been considered in the framework of the TFC combustion model. This effect is not connected with same specific modification of the turbulent structure due to combustion (the mixing of products and fresh mixture which results in increase of the flame brush width has a "gradient" nature) but with the fact that the progress variable turbulent transport is caused not only by turbulent diffusion but also directly by combustion.

The physical reason of this phenomenon is connected with gas expansion due to combustion. We have used here the simple upper bound estimation of this phenomenon that gave an opportunity to evaluate the "countergradient diffusion" term in Moreau experiments. In the TFC combustion model the transport term describes only a part of the transport, namely physical "gradient" diffusion which is responsible for the flame brush width development of the ISP flames (and the transport of any passive addition); the remaining "countergradient" part of the turbulent transport is incorporated into the model source term, i. e. the "countergradient diffusion" plus the chemical source term are modelled as a single term in the TFC progress variable equation. Good agreement with the standard experimental data and reasonable from physical point of view prediction of the space distribution of the combustion heat release and the counter-gradient diffusion phenomenon encourage us to use this model for the prediction of industrial premixed combustion.

\section{References}

[1] Zimont, V.L. (1977), "On the calculation of the partially premixed turbulent combustion 
of gases". In: Proceedings of Combustion of heterogeneous and gas systems, USSR Academy of Science, Chernogolovka (in Russian), pp. 76-80.

[2] Zimont, V.L. (1979), "The theory of the turbulent combustion at high Reynolds numbers", Combust. Expl. and Shock Waves, Vol. 15, pp. 305-311.

[3] Zimont, V.L., and Lipatnikov, A.N. (1994), Proceedings of the Academy of Sciences (Doklady Physical Chemistry), New York, 1994, pp. 440-443.

[4] Zimont, V.L., and Lipatnikov, A.N. (1995). "A numerical model of premixed turbulent combustion of gases", Chem. Phys. Reports, Vol. 14, n.7, 993-1025.

[5] Moss, J.B. (1980),"Simultaneous measurements of concentration and velocity in a open premixed turbulent flame", Combust. Science and Technology, Vol. 22, pp. 115-.

[6] Li, S.C., Libby, P.A., and Williams, F.A. (1994). "Experimental investigation of a premixed flame in an impinging turbulent stream", Twenty-fifth Symposium (International) on Combustion/ The Combustion Institute, pp. 1207-1214.

[7] Bray, K.N.C., Libby, P.A., Masura Goro, and Moss, J.B. (1981). "Turbulent production in turbulent premixed flames", Combust. Science and Technology, Vol. 25, pp. 127-140.

[8] Anand, M.S., and Pope, S.B. (1984). "Calculation of Turbulent Premixed Flames by PDF Methods", Combustion and Flame, Vol. 67, pp. 127-142.

[9] Moreau, P. (1977) 'Turbulent Flame Development in a High Velocity Premixed Flow', AIAA paper 77/49, 15th Aerospace Science Meeting.

[10] Bray, K. N. C., and Moss, J. B. (1977) "A Unified Statistical Model of the Turbulent Premixed Flame", Acta Astronautica, Vol. 4, p. 291-.

[11] Bray, K. N.C., and Libby, P. A. (1977) "Interaction Effects in Turbulent Premixed Flames", Physics of Fluids, Vol. 19, p. 1687-.

[12] Kollmann, W., and Janicka, J. (1982) "The probability density function of a passive scalar in turbulent shear flows", Combustion and Flame, Vol. 44, pp. 319-336. 
[13] Biagioli, F., (1997). "Comparison between presumed and Monte Carlo probability density function combustion modelling", Journal of Propulsion and Power, Vol. 13, No. 1.

[14] Pope, S. B. (1985) 'PDF methods for turbulent reactive flows', Prog. Energy Combust. Sci., Vol. 11, pp. 119-,

[15] Candel, S. M., and Poinsot, T. J. (1990) 'Flame stretch and the balance equation for the flame area', Combust. Sci. and Technol., Vol. 70, pp. 1-15.

[16] Borghi, R., (1985). R. Recent Advances in Aerospace Sciences, Pergamon, pp 117-138.

[17] Dinkelacker, F., Soika, A., Most, A., Höller, A. and Leipertz, A., (1998) "Measurement of local temperature gradients in turbulent combustion systems using two-dimentional laser diagnostics" 11th International Heat Transfer Conference, Kyongju, Korea 23. -28. 8. 1998

[18] Chen, Y., and Mansour, M.S., Twenty-Seventh Symposium (Int.) on Combustion, The Combustion Institute, Pittsburg, PA, 1998.

[19] O'young, F. and Bilger, R.W., "Scalar Gradient and Related Quantities in Turbulent Premixed Flames", (1997). Combustion and Flames, Vol. 109, pp. 682-700.

[20] Damköhler, G., (1947), NACA Tech. Memo. p. 1112 (in Russian).

[21] Shchelkin, K.I., (1947), NACA Tech. Memo. p. 1110 (in Russian).

[22] Zel'dovich, Ya.B., Barenblatt, G.I., Librovich, V.B., and Makhviladze, G.M., (1985). The Mathematical Theory of Combustion and Explosions, Plenum Publishing Corporation, New York.

[23] Maciocco, L., and Zimont, V. L., (1997). In: 20-th Annual Meeting of the Italian Section of the Combustion Institute "Frantic '97", pp X-2.1 - 2.4.

[24] Zimont, V., Polifke, W., Bettelini, M., and Weisenstein, W., (1998). Journal of Engineering for Gas Turbines and Power (Transactions of the ASME) Vol. 120, pp. 526-532. 
[25] Karpov, V.P., Lipatnikov, A.N. and Zimont V.L., (1994). Archivum Combustionis Vol. 4, No. 3-4, pp. 125-141.

[26] Karpov, V. P., Lipatnikov, A. N., and Zimont V. L., (1996). Twenty-Sixth Symposium (Int.) on Combustion, The Combustion Institute, Pittsburg, PA, p 249.

[27] Bray, K.N.C., (1987), In: Complex chemical reaction systems, Mathematical Modelling and Simulation, Springer Verlag, Heidelberg, p356. 


\section{Figures captions}

Fig. 1. "Countergradient diffusion" term versus the Favre progress variable $\tilde{c}$.

Fig. 2. Experimental set-up and inlet data.

Fig. 3. Contours of progress variable and stretch factor in the combustor.

Fig. 4. Profiles of $\mathrm{CH}_{4}$ (top) and $\mathrm{CO}$ (bottom) concentrations at several axial position in the combustor. Lines: predictions, Symbols: experiments.

Fig. 5. Profiles of temperature (top) and velocity (bottom) at several axial position in the combustor. Lines: predictions, Symbols: experiments.

Fig, 6 Contours and profiles of model and chemical source term for the progress variable.

Fig. 7 Contours of total, turbulent and "countergradient" fluxes of the progress variable in the combustor.

Fig. 8 Profiles of total, turbulent and "countergradient" fluxes of the progress variable at cross sections shown in figure 6 . 


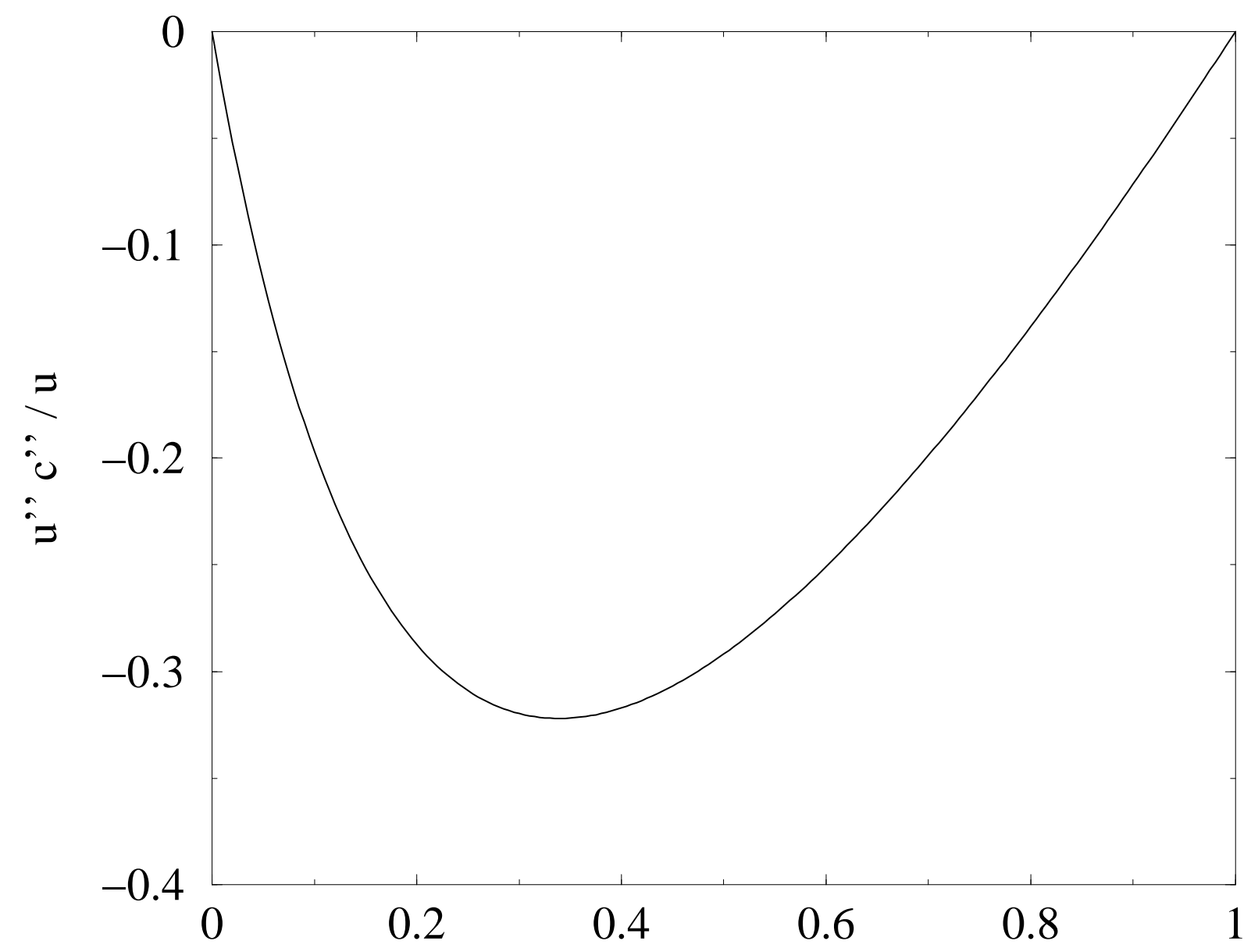

Favre progress variable, c

Figure 1: 


$\begin{array}{llll}\text { premixed reactants } & & \\ \Phi=0.8 & & \\ \mathrm{U}=65 \mathrm{~m} / \mathrm{s} & & \\ \mathrm{u}=8 \mathrm{~m} / \mathrm{s} \\ \mathrm{l}=0.024 \mathrm{~m} \\ \mathrm{~T}=600 \mathrm{~K}\end{array}$

Figure 2: 

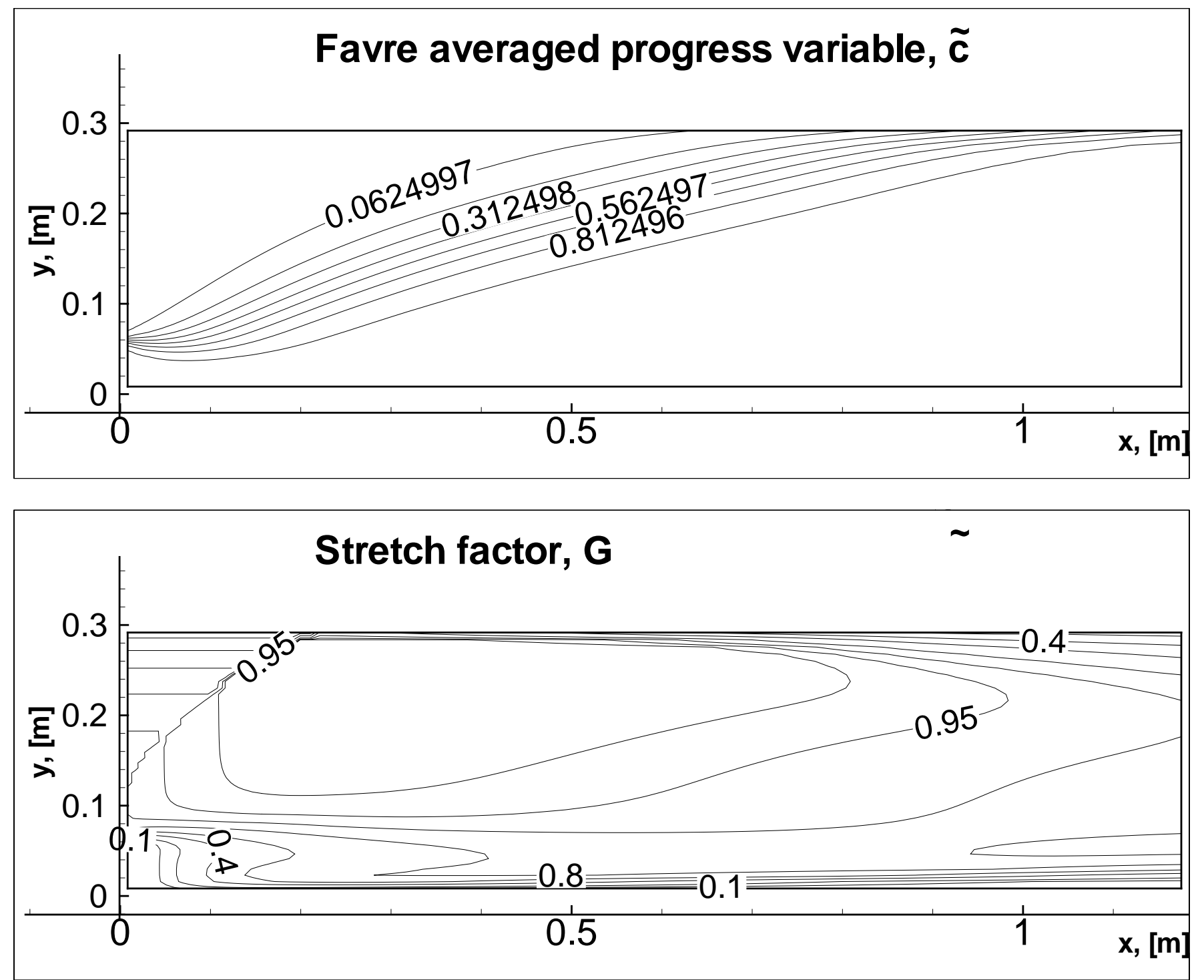

Figure 3: 

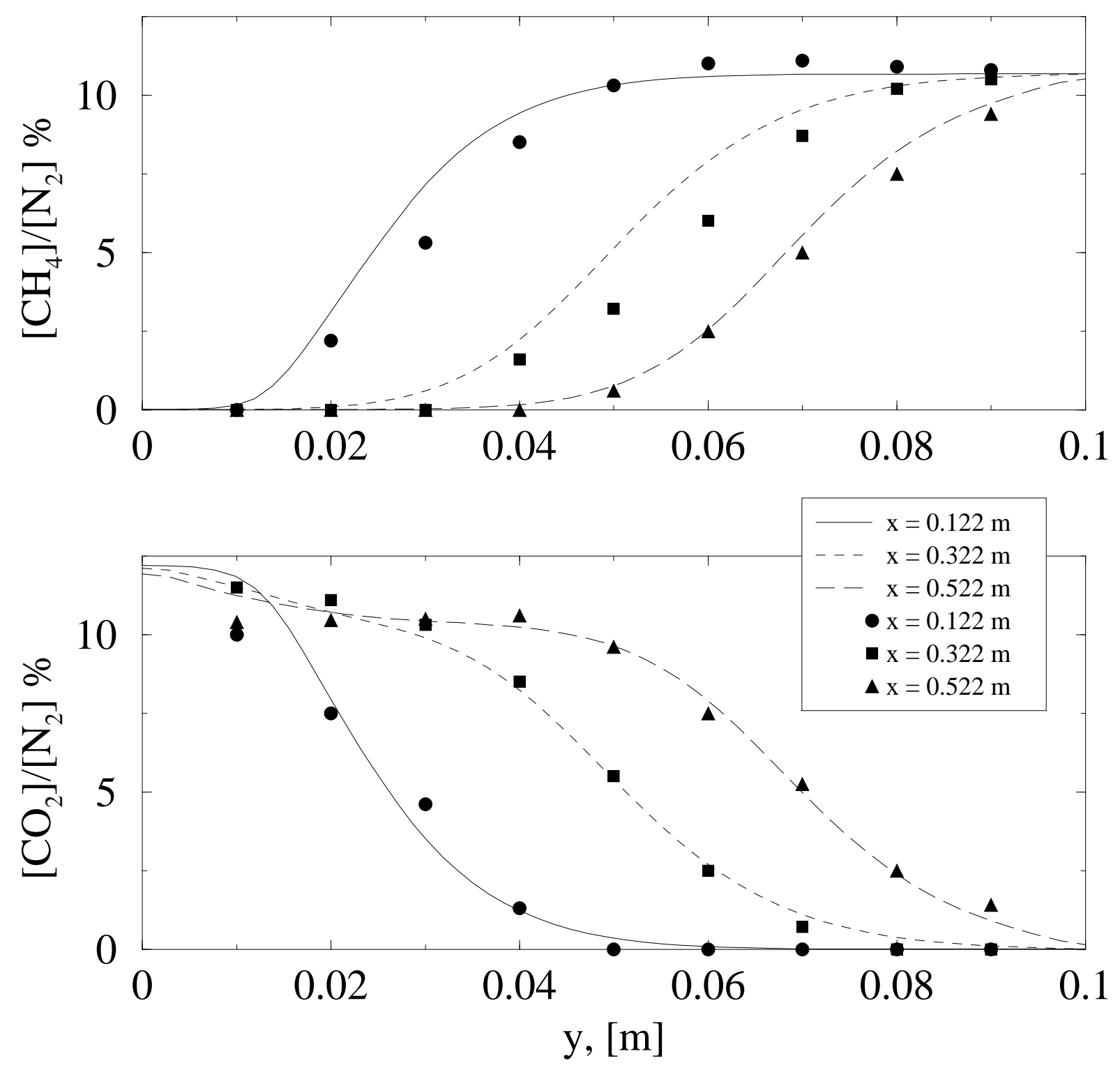

Figure 4: 


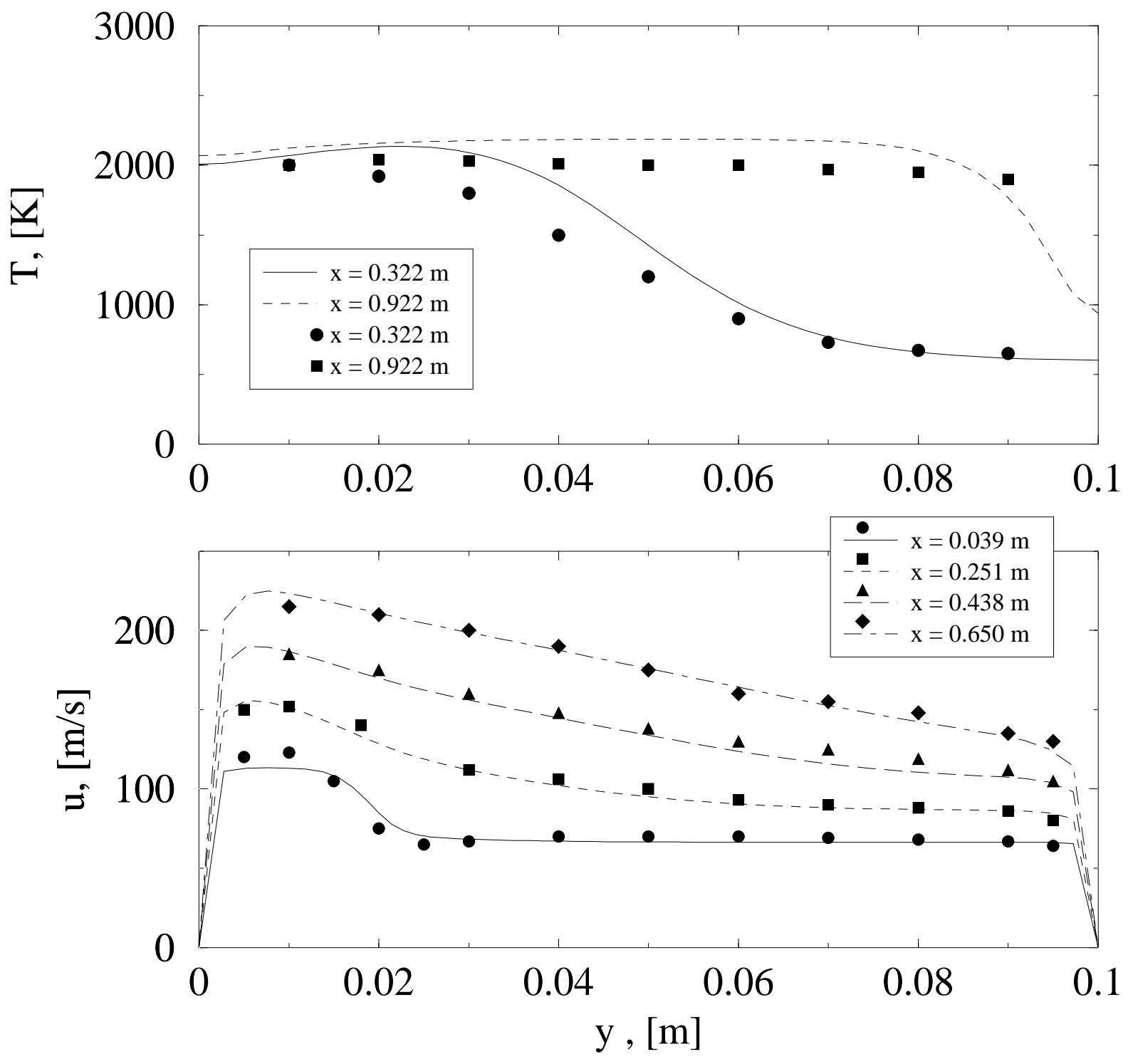

Figure 5: 

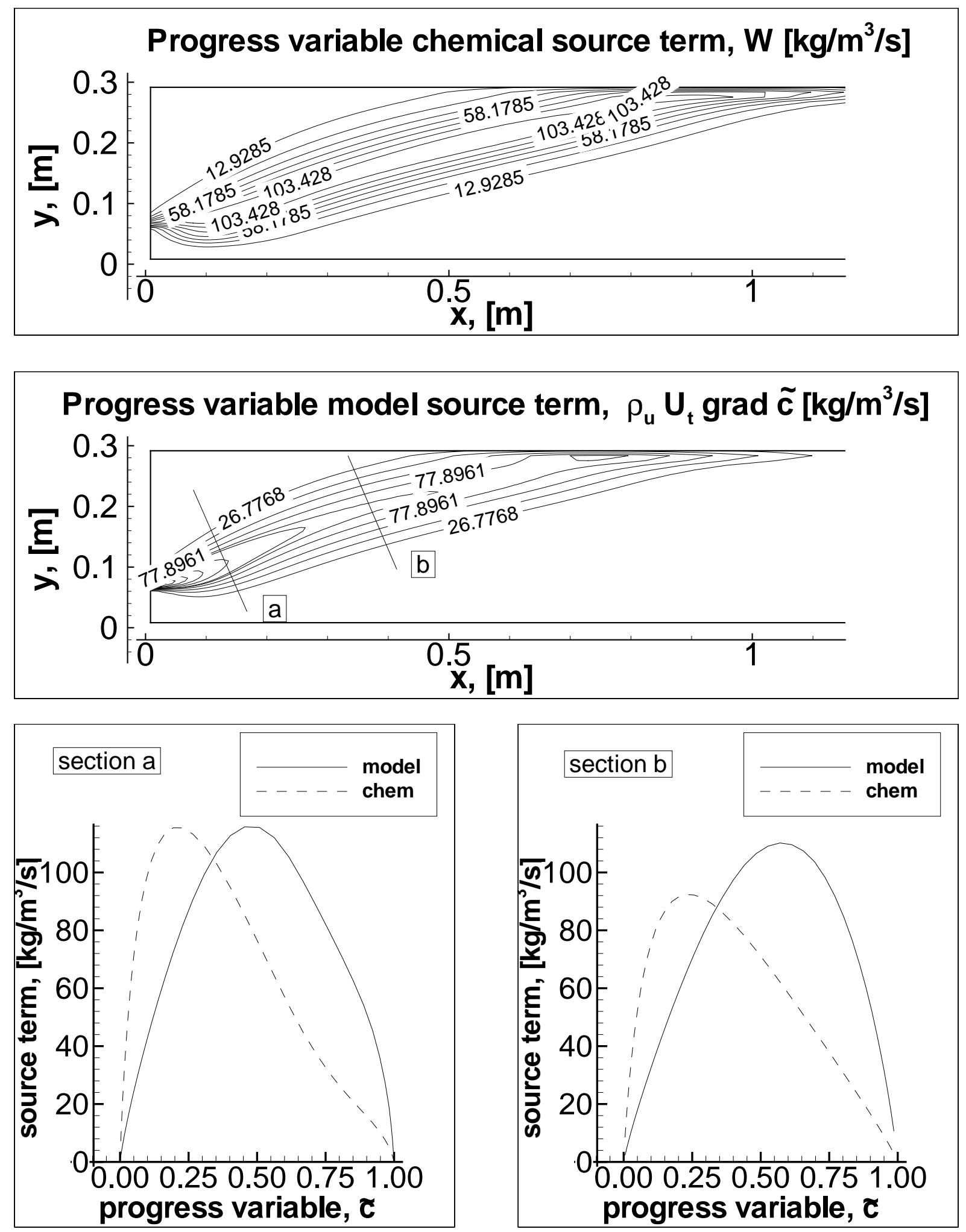

Figure 6: 

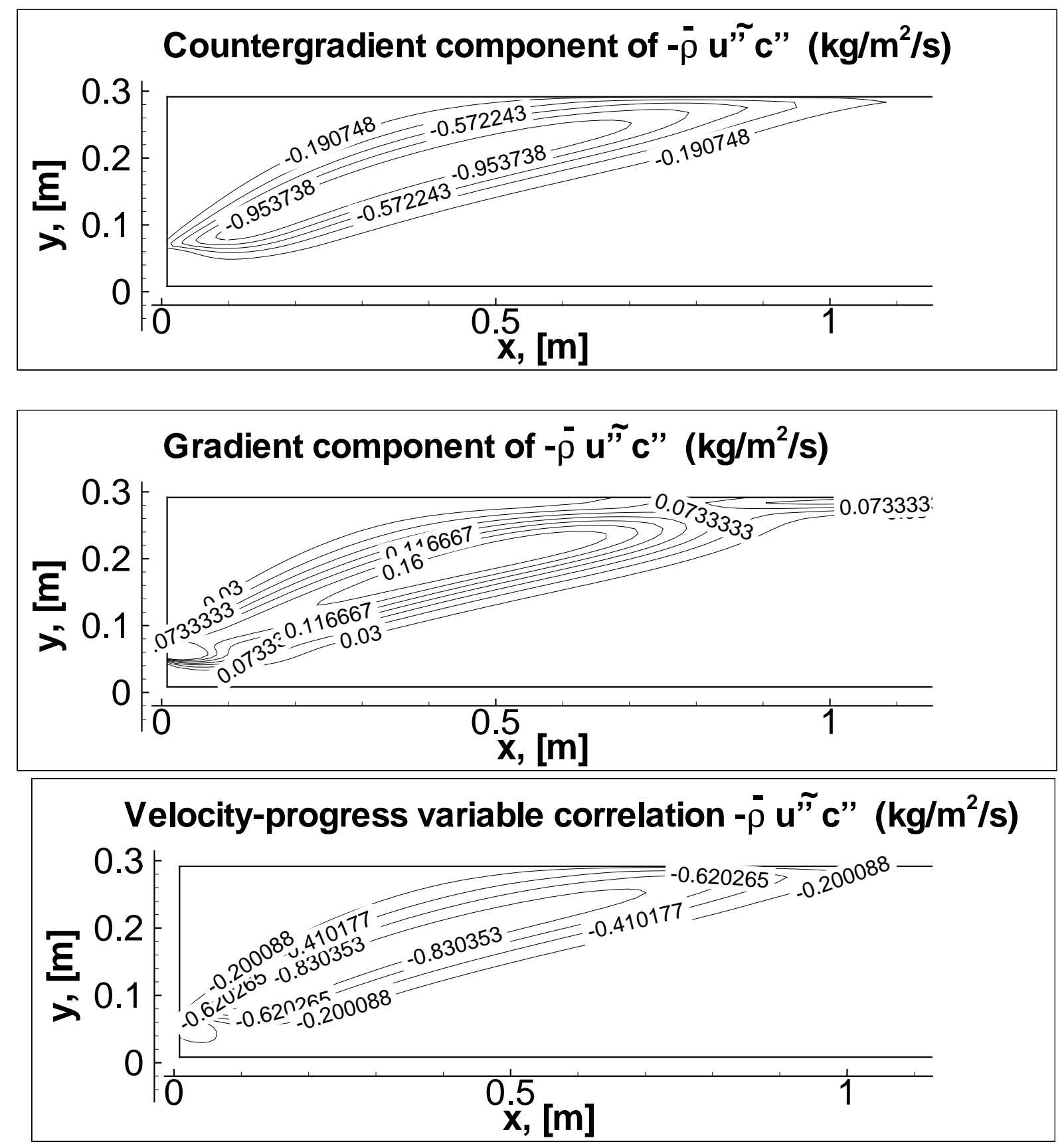

Figure 7: 

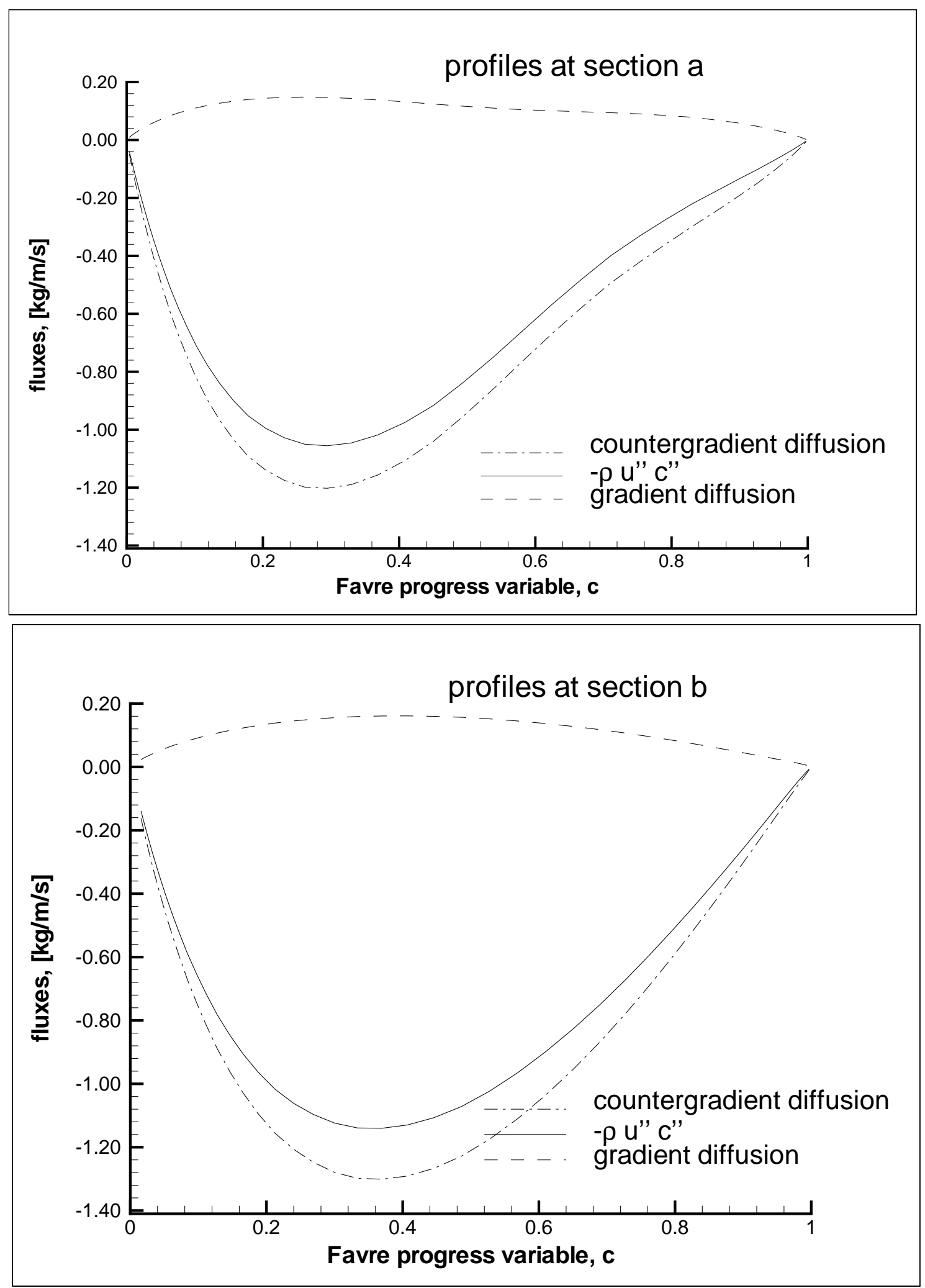

Figure 8: 